investigation and follow up for patients with fever and nonspecific symptoms are adequate to ensure timely diagnosis of PIMS-TS.

\section{CONCEPT OF NEUROPROTECTIVE NICU}

Mohammed Gaffari, Praney Jindal, Jisha Jeri, Shihab Kannappillil. Qatar

\subsection{6/bmjpo-2021-RCPCH.19}

Background Neurodevelopmental outcomes are of paramount importance for every clinician as the survival rates of term and preterm babies have continued to improve. In NICU every intervention should be aimed at either promoting the neurodevelopment or prevent any further insult to a premature brain. We aim to provide a framework for developing a Neuroprotective strategy for the Neonatal Intensive Care Unit (NICU) by describing five main domains below.

Objectives We achieve this in our NICU by a multi-disciplinary team consisting of neonatologists, respiratory therapists, occupational therapists, physiotherapists, social workers, pharmacists, and a dietician. This approach needs to be individualised for each unit based on the resources and services available.

Methods

Results

Conclusions Neuro assessment: clinical neuro assessment remains the most important tool, with strong predictive value for long-term outcomes. It is important to develop other tools of assessment like comfort and pain scoring. We use comfort Neo-scale as standard care. Neuroimaging is another important factor in the assessment. We have an agreed guideline to decide the frequency and the timing of the neuroimaging like cranial ultrasound and MRI.

Neuroprotection: Antenatal magnesium sulfate and antenatal steroids have become an established practice in most units ${ }^{1,2}$. Interventions like total body cooling have significantly improved the outcomes for babies with Hypoxic-ischemic injury. Optimal nutrition is another important element for the developing brain. We developed neonatal nutritional guidelines in collaboration with the clinical pharmacist and a dietician. Introduction of starter parenteral nutrition bags for out of hours use and having evidence-based feeding guidelines are known to improve the outcomes. We practice the golden hour protocol for all babies born before 28 weeks gestation and have introduced intra ventricular haemorrhage (IVH) prevention bundles ${ }^{3}$ for the same cohort of babies. Even though individual components of these bundles do not have strong evidence, there is some benefit when these interventions are offered as a bundle. Our care bundle involves midline positioning, using log roll, minimal handling, maintaining normothermia, avoiding IV boluses, and maintaining normal $\mathrm{CO} 2$ levels

Neuromonitoring: Tools like an amplitude-integrated electroencephalogram (aEEG), near-infrared spectroscopy (NIRS), and onsite MRI are gaining popularity. eEEG should be routinely used in Hypoxic Ischemic Encephalopathy (HIE) babies when available. All team members should be trained in its application and interpretation. Near Infra-Red Spectroscopy is a developing modality used by few units to monitor cerebral oxygenation. We have recently started to pilot these machines.

Neurodevelopment: The environment of the NICU has been shown to affect the developing brain. Strategies should be developed to optimise the sleep of the baby by reducing lighting and noise levels. We use positioning tools like boundaries and midliners as part of their neuro development.

Neuro intervention: We use therapeutic techniques like auditory, tactile, visual, and vestibular (ATVV) stimulation ${ }^{4}$. It is an evidence-based technique used to increase alertness in medically stable preterm infants. We use Prechtl's Qualitative Assessment of General Movements observational tool ${ }^{5}$. It is the most predictive tool $(98 \%$ sensitivity) for detecting cerebral palsy. This helps provide targeted treatment at an earlier stage.

\section{ASSESSING THE EFFECTIVENESS OF NUTRITION- FOCUSSED WORKSHOPS IN SUPPLEMENTING PRIMARY SCHOOL EDUCATION}

Asvini Balasubramaniam, Naomi Richardson, Karishma Tailor, Anmol Landa, Jonti Cole, Rania Khan. UK

\subsection{6/bmjpo-2021-RCPCH.20}

Background Over 340 million young people aged 5-19 were overweight or obese in 2016 (WHO, 2020). Educational interventions implemented during childhood may be key to ameliorating the burden of obesity on global health (Llargues et al., 2011). The Food for Thought Project was a volunteering scheme, led by medical students, aimed at further educating primary school children about nutrition. Interactive workshops were conducted for children to explore healthy food alternatives, nutritional labels, sugar content, and diabetes.

Objectives The primary objective was to assess the effectiveness of using nutrition-focussed workshops alongside the existing curriculum, emphasising the importance of healthy eating and diabetes. The secondary objective was to provide teaching opportunities to medical students with an interest in paediatrics and lifestyle medicine.

Methods Quantitative and qualitative data was collected from pupils, teachers, and medical students. Pupils $(n=106)$ in Years 4 and 5 filled out a questionnaire before and after the session, self-evaluating their ability to choose healthy foods, read nutrition labels, and describe diabetes. The questionnaire used a 3-point scale of 'Yes', 'A Little', and 'No'. Additionally, the children rated their overall experience of the workshop using a pictorial 5-point scale of 'Very Bad' to 'Brilliant'. To further assess the impact of the workshop, teachers $(n=11)$ were given a questionnaire that employed a 5-point Likert scale, obtaining feedback on the relevance and appropriateness of the content for the age group. To address the secondary objective, qualitative feedback was obtained from medical students $(n=11)$ to assess what they enjoyed and gained from leading the workshops.

Results As illustrated in table 1, the number of pupils who selected 'Yes' on the questionnaire increased in all three parameters after the session. This demonstrated the workshop was able to increase the number of students who felt confident in their knowledge of the content delivered. When asked to rate the session, $85 \%$ of students responded with 'Brilliant' and 9\% responded with 'Very Good'. Additionally, all teachers either 'Strongly Agreed' or 'Agreed' that the workshop was relevant and appropriate for the age group and were interested in having further sessions. Furthermore, medical student feedback responses indicated the project was a great 
Abstract 28 Table 1 The proportion of pupils $(n=106)$ that selected 'Yes' on the questionnaire

\begin{tabular}{llll}
\hline & $\begin{array}{l}\text { Before the Session } \\
\text { (\% of children) }\end{array}$ & $\begin{array}{l}\text { After the Session } \\
\text { (\% of children) }\end{array}$ & $\begin{array}{l}\text { Percentage } \\
\text { Change (\%) }\end{array}$ \\
\hline $\begin{array}{l}\text { Do you know how to } \\
\text { choose healthy foods? }\end{array}$ & 65 & 89 & +24 \\
$\begin{array}{l}\text { Do you know how to read } \\
\text { nutrition labels? }\end{array}$ & 35 & 75 & +40 \\
$\begin{array}{l}\text { Do you know what } \\
\text { diabetes is? }\end{array}$ & 60 & 86 & +26 \\
\hline
\end{tabular}

opportunity to engage with children, practice public speaking skills, and gain confidence.

Conclusions Overall, the feedback received was positive and encouraging. The results show the workshops have successfully engaged with pupils, creating an exciting environment to learn about nutrition. Ultimately, early encouragement of healthy lifestyle choices in childhood may be critical to the development of healthier long-term habits (Llargues et al., 2011). In conclusion, the Food for Thought Project could be an effective school-based intervention to help combat childhood obesity and other lifestyle diseases that may develop later in life.

\section{MULTISYSTEM INFLAMMATORY SYNDROME IN CHILDREN (MIS-C/PIMS): AN OVERVIEW}

Eunice Jia Lin Tang. UK

\subsection{6/bmipo-2021-RCPCH.21}

Background Multisystem inflammatory syndrome in children (MIS-C) or Paediatric inflammatory multisystem syndrome is a newly emerged hyper-inflammatory syndrome that is associated with SARS-CoV-2 infection in the paediatric population.

Objectives We aim to identify commonly presented clinical features, imaging findings, laboratory findings, treatment modalities and clinical outcomes of MIS-C/PIMS. We also aim to compare Kawasaki Disease and MIS-C/PIMS for better identification and management of these patients.

Methods A systematic review was conducted from 1st December 2019 to 30th August 2020. Three medical databases (PubMed, Ovid Resources and the WHO COVID-19 database) were included in this study. Inclusion criteria were all observational studies, case reports or case series that reported data on MIS-C or PIMS.

Results We yielded 48 studies $(\mathrm{N}=1604)$ from 12 countries. Median age ranged from 2 to 19.9 years. $88 \%$ had positive SARS-CoV-2 PCR or serology tests. Reported clinical features include fever (100\%), gastrointestinal symptoms (87\%), rash (56\%), conjunctivitis (48\%) and shock (47\%). ECHO abnormalities $(\mathrm{N}=471)$ were most commonly reported. Laboratory findings include elevated inflammatory markers (66\%-95\%), deranged LFTs (54\%) and cardiac biomarkers (49\%-55\%). Common treatment choices: intravenous immunoglobulin (76\%), steroids (59\%) and aspirin (33\%). ICU admission rate was $72 \%$ and mortality rate was $2 \%$.

Conclusions MIS-C/PIMS is an immune-mediated complication associated with COVID-19. Clinical manifestation vary and majority presents with evidence of multiorgan dysfunction. Close monitoring and high level of care should be given to suspected or confirmed cases. Supportive care remains the mainstay treatment. While manifestation of MIS-C/PIMS overlaps with $\mathrm{KD}$, it is a distinct entity based on their differences in age distribution, geographical and racial variation, laboratory findings, associated features and clinical outcomes.

\section{HIGH FLOW NASAL CANNULA OXYGEN USE IN A DISTRICT GENERAL HOSPITAL IN THE PRE-COVID ERA FROM OCTOBER 2019 TO APRIL 2020}

Laura Johnston, Laura Johnston, Lauren Hetherington, Veena Vasi. UK

\subsection{6/bmjpo-2021-RCPCH.22}

Background High Flow Nasal Cannula (HFNC) oxygen is used to deliver heated and humidified mixture of air and oxygen at a flow higher than the patient's inspiratory flow. It reduces respiratory distress and mouth dryness. The greater oxygen flow 'washes out' the end expiratory oxygen depilated gas, meaning with the next breath, the patient inhales more oxygen. This dead space wash out also reduces $\mathrm{CO} 2$ rebreathing. Reductions in rates of intubation in infants with bronchiolitis have also been reported following introduction of HFNC therapy; however research remains limited within paediatrics and it has been used in patients with conditions, other than bronchiolitis. In our District General Hospital (DGH), we have a guideline for use of HFNC in patients with bronchiolitis but not for use in other respiratory disease. The use of HFNC for the latter group is at individual consultant discretion after discussion with Paediatric Intensive Care Objectives

- What were the incidence, indication, demographics, duration and outcome of patients who received HFNC therapy on the Children's ward in Craigavon Hospital over a 6 month period from October 2019 to March 2020?

- When patients received HFNC therapy outside our current indication, were they discussed with PICU in terms of suitability to initiate or continue therapy in the children's ward?

- Was there a delay or perceived delay in escalation of treatment to tracheal intubation in those who had treatment failure on HFNC?

Methods This was a retrospective chart review. Patient demographics, indication, duration of treatment and outcome are recorded in a pre-designed proforma

SHSCT Research Governance team advised this is a service evaluation quality improvement which did not require ethics approval (Appendix 2).

Treatment success was defined as 'patient improving on HFNC with successful weaning of respiratory support.' Treatment failure was described as the 'patient deteriorating despite treatment and requiring admission to PICU'

Results are tabulated in XCEL format, analysed and conclusions drawn with recommendations

Results

- 28 children required HFNC therapy over a 6 month period with peak activity in November 2019

- Male to female ratio was 3.5:1

- Commonest indication for use (85\% cases) was bronchiolitis

- Comorbidities were seen in 50\% of the cases with commonest being prematurity

- All patients receiving HFNC outside agreed indications were appropriately discussed with PICU 